Published in final edited form as:

Int J Cardiovasc Imaging. 2012 February ; 28(2): 285-293. doi:10.1007/s10554-011-9811-8.

\title{
Acute infarct selective MRI contrast agent
}

\section{Robert Kirschner,}

Department of Biochemistry and Molecular Genetics, University of Alabama at Birmingham, MCLM 556, Birmingham, AL 35294-0005, USA

Heart Institute, Faculty of Medicine, University of Pecs, Pecs, Hungary

Elgavish Paramagnetics Inc., Birmingham, AL, USA

\section{Akos Varga-Szemes,}

Department of Biochemistry and Molecular Genetics, University of Alabama at Birmingham, MCLM 556, Birmingham, AL 35294-0005, USA

Heart Institute, Faculty of Medicine, University of Pecs, Pecs, Hungary

Elgavish Paramagnetics Inc., Birmingham, AL, USA

\section{Tamas Simor,}

Department of Biochemistry and Molecular Genetics, University of Alabama at Birmingham, MCLM 556, Birmingham, AL 35294-0005, USA

Heart Institute, Faculty of Medicine, University of Pecs, Pecs, Hungary

Elgavish Paramagnetics Inc., Birmingham, AL, USA

\section{Pal Suranyi,}

Department of Biochemistry and Molecular Genetics, University of Alabama at Birmingham, MCLM 556, Birmingham, AL 35294-0005, USA

Elgavish Paramagnetics Inc., Birmingham, AL, USA

\section{Pal Kiss,}

Department of Biochemistry and Molecular Genetics, University of Alabama at Birmingham, MCLM 556, Birmingham, AL 35294-0005, USA

Elgavish Paramagnetics Inc., Birmingham, AL, USA

\section{Balazs Ruzsics,}

Department of Biochemistry and Molecular Genetics, University of Alabama at Birmingham, MCLM 556, Birmingham, AL 35294-0005, USA

Elgavish Paramagnetics Inc., Birmingham, AL, USA

\section{Brigitta C. Brott,}

(c) Springer Science+Business Media, B.V. 2011

Correspondence to: Gabriel A. Elgavish, gabi@uab. edu.

Conflict of interests Dr. Kirschner and Dr. Varga-Szemes are employees, P. Kiss, P. Suranyi and B. Ruzsics were employees, and Drs. A. Elgavish and G. A. Elgavish are officers, of Elgavish Paramagnetics Inc., as well as employees of UAB. 
Division of Cardiovascular Disease, Department of Medicine, University of Alabama at Birmingham, Birmingham, AL, USA

Ada Elgavish, and

Division of Clinical Immunology and Rheumatology, Department of Medicine, University of Alabama at Birmingham, Birmingham, AL, USA

Elgavish Paramagnetics Inc., Birmingham, AL, USA

\section{Gabriel A. Elgavish}

Department of Biochemistry and Molecular Genetics, University of Alabama at Birmingham, MCLM 556, Birmingham, AL 35294-0005, USA

Elgavish Paramagnetics Inc., Birmingham, AL, USA

Gabriel A. Elgavish: gabi@uab.edu

\section{Abstract}

To determine the infarct affinity of a low molecular weight contrast agent, Gd(ABE-DTTA), during the subacute phase of myocardial infarct (MI). Dogs $(n=7)$ were examined, using a closed-chest, reperfused MI model. MI was generated by occluding for 180 min the Left Anterior Descending (LAD) coronary artery with an angioplasty balloon. DE-MRI images with Gd(ABEDTTA) were obtained on days 4, 14, and 28 after MI. Control DE-MRI by Gd(DTPA) was carried out on day 27. T2-TSE images were acquired on day 3,13 and 27. Triphenyltetrazolium chloride (TTC) histomorphometry validated postmortem the existence of infarct. Gd(ABE-DTTA) highlighted the infarct on day 4 , but not at all on day 14 or on day 28 , following MI. On day 4 , the mean \pm SD signal intensity (SI) of infarcted myocardium in the presence of Gd(ABE-DTTA) significantly differed from that of healthy myocardium ( $45 \pm 6.0$ vs. $10 \pm 5.0, P<0.05)$, but it did not on day $14(11 \pm 9.4$ vs. $10 \pm 5.7, \mathrm{P}=\mathrm{NS})$, nor on day $28(7 \pm 1.5$ vs. $7 \pm 2.4, \mathrm{P}=\mathrm{NS})$. The mean \pm SD signal intensity enhancement (SIE) induced by Gd(ABE-DTTA) was $386 \pm 165 \%$ on day 4, significantly different from mean SIE on day $14(9 \pm 20 \%)$, and from mean SIE on day 28 $(12 \pm 18 \%)$, following MI $(P<0.05)$. The last two mean values did not differ significantly $(\mathrm{P}=$ NS) from each other. As control, Gd(DTPA) was used and it did highlight the infarct on day 27, inducing a mean SIE value of $312 \pm 40 \%$. The mean SIE on day 3, 13, or 27 did not vary significantly ( $\mathrm{P}=\mathrm{NS})$ on the T2-TSE images $(114 \pm 41 \%, 123 \pm 41 \%$, and $150 \pm 79 \%$, respectively). Post mortem, the existence of infarcts was confirmed by TTC staining. The infarct affinity of $\operatorname{Gd}(A B E-D T T A)$ vanishes in the subacute phase of scar healing, allowing its use for infarct age differentiation early on, immediately following the acute phase.

\section{Keywords}

Myocardial infarct; Magnetic resonance imaging; Delayed enhancement; Contrast agent

\section{Introduction}

Differentiation between acute and older MIs is of great importance in clinical decisionmaking. There are several clinical scenarios where differentiation between acute and older myocardial infarct may be crucial, such as exclusion of myocardial reinfarction in the 
presence of an old infarct, or localization of the "culprit" vessel in patients with multi-vessel CAD in acute Non-ST-segment-elevation-MI (NSTEMI) in the presence of an old MI.

In the last decade, delayed enhancement (DE) inversion recovery gradient echo (IR-GRE) MRI with standard extracellular contrast agents became the most important and accurate imaging tool for assessing either the localization, the transmurality, or the extent, of MIs [1]. $\mathrm{DE}$ is also capable of differentiating stunned myocardium from necrotic tissue in the acute phase [2], and hibernated myocardium from scar tissue in the chronic phase, of a MI [3]. A significant shortcoming of this method is, however, that standard extracellular contrast agents used with DE-MRI highlight both acute and chronic MI. Also, the magnitude of signal intensity enhancement in the territory of MI in the two stages is similar [2, 4].

We have shown $[5,6]$ that a low molecular weight MRI contrast agent developed in our laboratory, Gd(ABE-DTTA), induces a DE effect in acute (four-day old), but not in late subacute (four-week old), MI in a canine, double infarct model. The infarct affinity of Gd(ABE-DTTA), however, is not known between days 4 and 28 following MI. The purpose of the current study has been the determination of this affinity in the subacute phase of scar healing, and to compare it to the already known affinity during the late subacute phase. For the purpose of this comparison, a different MI model and experimental design were applied compared to those previously used $[5,6]$. In the present study a single myocardial infarct was generated and followed longitudinally, and determination of the contrast agent's affinity was extended to day 14. This design allowed the comparison of the SIE produced by Gd(ABE-DTTA) on day 4 to those on day 14 as well as on day 28 .

Gd(ABE-DTTA) is in investigational phase, and has no deleterious physiological effects [7]. Its clearance from the blood has a kinetics similar to that of blood pool contrast agents, although it also displays partly extracellular characteristics [8]. It demonstrates high affinity for acute MI [8], with slow, distinctive tissue-persistent kinetics. Gd(ABE-DTTA) is taken up into, and is washed out from the acutely infarcted tissue slowly, in contradistinction from the kinetics of standard extracellular agents' uptake and wash-out. This CA attains its maximum concentration in the acutely infarcted tissue around $48 \mathrm{~h}$ after administration, but a useful contrast is already achieved at $24 \mathrm{~h}$. The contrast remains detectable in the infarct for a few days [8]. Gd(ABE-DTTA) quantifies the extent of acute MI accurately $[9,10]$.

We hypothesized that Gd(ABE-DTTA) differentiates similarly between acute and 2-weekold MI as it does between acute and 4-week old MI, i.e. the infarct affinity of Gd(ABEDTTA) vanishes already in the subacute phase of scar healing.

\section{Methods}

\section{Gd(ABE-DTTA) sample preparation and administration}

Gd(ABE-DTTA) was synthesized, and samples were prepared, as described by Saab et al. [11]. The in vitro relaxivity was measured for every sample [11] to guarantee consistent quality of agent sample before administration. Gd(ABE-DTTA) was administered at the dose of $0.05 \mathrm{mmol} / \mathrm{kg} 24 \mathrm{~h}$ before every Gd(ABE-DTTA)-enhanced DE-MRI session. This dose was four-fold lower than the typical dose $(0.2 \mathrm{mmol} / \mathrm{kg})$ at which Gd-DTPA had been 
used for DE-MRI, yet induced an in vitro relaxation enhancement equivalent to that of the higher dose Gd-DTPA.

\section{Study design}

Dogs $(n=7)$ were studied in a longitudinal, closed-chest, reperfused, single MI model, as follows. MI was generated by occluding for $180 \mathrm{~min}$ the LAD coronary artery with an angioplasty balloon. Inversion-recovery fast gradient echo (IR-FGE) MRI images were obtained on days 4, 14, and 28 after MI, and delayed enhancement was generated with Gd(ABE-DTTA). On days 13 and 27 post MI, the same MRI IR-FGE image sequence was used without contrast agent, to demonstrate that the previously administered tissue-persistent CA, Gd(ABE-DTTA), totally cleared from the myocardial infarct as judged by comparison to precontrast images. In addition, control visualization of the infarct was carried out on day 27 with Gd(DTPA) (standard extracellular contrast agent), to ascertain that the infarct was still in place despite the fact that the acute-infarct specific agent did not highlight it. To avoid a potential interaction of the two CAs, Gd(ABE-DTTA) was administered on day 27 only upon the complete clearing of Gd(DTPA). T2-weighted Turbo Spin Echo (T2-TSE) images were acquired on day 3,13 and 27 before contrast agent administration. Outline of the experimental design is shown in Fig. 1.

\section{Surgical procedure}

The animal protocol was approved by the IACUC of our institution in full compliance with the 'Guidelines for the Care and use for Laboratory Animals' (NIH). Seven male hounds $(18-20 \mathrm{~kg}$ ) were used. Twelve hours prior to procedure food was taken away and $325 \mathrm{mg}$ Aspirin given. Hounds were anesthetized with a Ketamine $(5.0 \mathrm{mg} / \mathrm{kg})$ and Diazepam $(0.5$ $\mathrm{mg} / \mathrm{kg}$ ) mixture, intubated, and connected to a Hallowell EMC Model 2000 respirator (Pittsfield, MA, USA) operated with a tidal volume of $400 \mathrm{ml}$ at a rate of 16 BPM. Anesthesia was maintained by continuous flow of Isoflurane (2.5-3 volume \%), and repeated Fentanyl (50-100 ug I.V. every $30 \mathrm{~min}$ ) administration. ECG electrodes were placed on the chest to record electrophysiological signs of myocardial ischemia and arrhythmias. Heart rate and blood oxygen saturation were monitored using a pulse-oxymeter with the probe placed on the animal's tongue. The left femoral artery was separated surgically and an arterial sheath (6-8 French) was inserted. An I.V. line was placed to administer infusion and drugs. Heparin $(100 \mathrm{IU} / \mathrm{kg}$ ) was given intravenously to maintain the activated clotting time (ACT) above $300 \mathrm{~s}$. A properly sized 2-3 mm angioplasty balloon was introduced into the LAD under fluoroscopic guidance and inflated for 180 min to create MI. Thereafter, the balloon was deflated to restore coronary circulation. Fluoroscopic coronary angiography confirmed the onset of reperfusion after balloon deflation.

On day 3, 4, 13, 14, 27, and 28 following MI, animals were re-anesthetized as described above, and MRI studies performed. After the last MRI session, animals were sacrificed, hearts were excised and sliced (5 mm thick slices). TTC staining $\left(2 \%, 37^{\circ} \mathrm{C}\right)$ was carried out to validate the existence of myocardial infarcts. Both sides of each TTC slice were digitally photographed. 


\section{Magnetic resonance imaging}

A 1.5T GE Signa-Horizon CV/i scanner (Milwaukee, WI, USA) was used. A cardiac phased-array coil and ECG gating were employed. Breath-hold was performed using a manual switch on the respirator at end-expiration. A $180^{\circ}$-prepared, segmented, inversionrecovery fast gradient-echo (IR-FGE) pulse sequence was used with: Field of View (FOV) of $30 \mathrm{~cm}$, Echo Time (TE) of $3.32 \mathrm{~ms}$, Views per Segment of 16, Shot Length of $114.8 \mathrm{~ms}$, Repetition Time (TR) two cardiac cycles (1100-1600 ms), slice thickness of $10 \mathrm{~mm}$, Image Matrix of $256 \times 256$, Flip Angle of $25^{\circ}$, NEX of 1 . The Inversion Time (TI) was optimized to null the signal in normal myocardium. Double inversion-recovery (black-blood) fast-spinecho images were generated at end-diastolic phase of the cardiac cycle with the following parameters: FOV of $30 \mathrm{~cm}$, TE of $60 \mathrm{~ms}$, Echo Train Length of 16, Shot Length of $140 \mathrm{~ms}$, TR of two cardiac cycles (1100-1600 ms), Slice Thickness of $10 \mathrm{~mm}$, Image Matrix of 256 $\times 256$, Flip Angle of $90^{\circ}, \mathrm{NEX}$ of 1 . Conventional cardiac angulation planes were set and short axis slices covering the entire LV were obtained (six slices per heart). The scan (breathhold at end-expiration) time per image was 15-20 s for DE-MRI or for T2w imaging. The total imaging time was around 6 min for both sequences.

\section{Contrast agent}

In the MRI session on day 13, the IR-FGE sequence was used without CA administration. On day 27, a $0.2 \mathrm{mmol} / \mathrm{kg} \mathrm{Gd(DTPA)} \mathrm{(Magnevist,} \mathrm{Schering,} \mathrm{Kenilworth,} \mathrm{NJ)} \mathrm{bolus} \mathrm{was}$ administered intravenously. DE images were acquired with the IR-FGE sequence 15-20 min thereafter. In the MRI sessions on days 4, 14, and 28, DE images were similarly obtained, 24 $h$ after i.v. administration of $0.05 \mathrm{mmol} / \mathrm{kg} \mathrm{Gd}(\mathrm{ABE}-\mathrm{DTTA})$.

\section{Image analysis}

MRI Dicom images were imported as image sequences with the use of ImageJ. In all images, the endo-, and epicardial contours were traced manually. Efforts were taken to avoid including any artificially high signal intensity (SI) due to inadequately suppressed slow flow within the left ventricular cavity space on T2w images. Large regions of interests, remote from the infarct, were selected to measure baseline SI \pm SD of the healthy myocardium. All other analyses were automated to eliminate observer bias. In accordance with the literature [12], pixels with SI above the mean $+6 \mathrm{SD}$ of the normal myocardium were regarded as "enhanced" pixels. A myocardial region was regarded as affected [13] when at least 10 connected pixels of the myocardium revealed enhanced signal intensity. Regions of interest (ROIs) were drawn automatically (by ImageJ) around all areas of the affected pixels in every infarct-affected slice, and their mean SI was measured. If no 10 connected enhanced pixels were found in any slice of a DE image set, an ROI ( 100 pixels) was placed in the center of infarcted (but unenhanced) myocardium based on TTC staining [14], and the mean SI was measured on the stored image. The mean percent SIE was computed by [15]:

$$
\mathrm{SIE}=100 \times \frac{\mathrm{SI}_{i}-\mathrm{SI}_{n}}{\mathrm{SI}_{n}}
$$


where $\mathrm{SI}_{i}$ and $\mathrm{SI}_{n}$ were the mean signal intensity in infarct and normal myocardium, respectively.

\section{Statistical analysis}

Statistical analysis was carried out by SigmaStat (Version 2.03; SPSS Inc, Chicago, IL, USA) and SPSS (Release 13.0; SPSS Inc, Chicago, IL, USA). Results are reported as mean \pm SD. One-way repeated measures analysis of variance was used to compare mean signal intensity enhancement (SIE) of DE images enhanced by Gd(ABE-DTTA) or by the T2 weighting sequence on the different days, since these data sets passed both the normality and the equality of variances tests. An overall significance $(P<0.05)$ was established for rejecting the null hypothesis that the three groups are not different, therefore pairwise differences between the groups were assessed by using the Holm-Sidak method of adjustment for multiple comparisons. Two-way repeated measures analysis of variance was used to compare the SI values among experimental groups (factor 1: infarct vs. normal, factor 2: age of infarct). Although the test of normality failed in this data set, thanks to the equality of variances, the equality of group sizes, and the high power of the performed test ( 0.989 with $\alpha=0.05)$, the assumption of the $\mathrm{F}$ test in the two-way ANOVA with repeated measures was not violated [16]. An overall significance $(P<0.05)$ was established for rejecting the null hypothesis that the six groups are not different. Pairwise differences between the groups were assessed by using the Holm-Sidak method of adjustment for multiple comparisons.

A value of $P<0.05$ was considered significant in all statistical tests.

\section{Results}

Gd(ABE-DTTA) highlighted the infarct in all cases on day 4, but not at all on day 14 or on day 28 following MI (see an example shown in Fig. 2). By days 13 and 27 post MI, the previously administered Gd(ABE-DTTA) completely cleared from the myocardium, including the infarct area. The mean \pm SD signal intensity (SI) of infarcted myocardium in the presence of Gd(ABE-DTTA) significantly differed on day 4 from that of healthy myocardium ( $45 \pm 16.0$ vs. $10 \pm 5.0, P<0.05)$, but it did not on day $14(11 \pm 9.4$ vs. $10 \pm$ 5.7, $\mathrm{P}=\mathrm{NS}$ ), nor on day 28 ( $7 \pm 1.5$ vs. $7 \pm 2.4, \mathrm{P}=\mathrm{NS}$ ) (Fig. 3 .). The mean $\pm \mathrm{SD}$ signal intensity enhancement (SIE) induced by Gd(ABE-DTTA) was $386 \pm 165 \%$ on day 4 , significantly different from SIE on day $14(9 \pm 20 \%)$, and on day $28(12 \pm 18 \%)$ following MI $(P<0.05)$. The last two mean values did not vary significantly $(\mathrm{P}=\mathrm{NS})$ between them. $\operatorname{Gd}(\mathrm{DTPA})$ highlighted the infarct in all cases on day 27, inducing a SIE value of $312 \pm$ 40\%. T2 weighted (T2w) signal enhancement was seen in the segments supplied by the infarct related artery (IRA) (Fig. 4). This enhancement appeared not only during the acute but also during the subacute and late subacute phase of the MI. The pattern of the enhancement was very similar in these three different phases of scar healing. The mean \pm SD SIE on day 3, 13, or 27 did not differ significantly ( $\mathrm{P}=\mathrm{NS}$ ) on T2-TSE images (114 \pm $41,123 \pm 41$, and $150 \pm 79 \%$, respectively). The existence of infarcts was also confirmed by TTC staining. 


\section{Discussion}

It had been shown earlier that Gd(ABE-DTTA) differentiates between acute and 4-week old myocardial infarcts (MI) in a canine, double infarct model $[5,6]$. This previous model was designed to demonstrate the agent's ability to differentiate between acute and late subacute MIs in an animal having both types of myocardial infarct simultaneously. The principal finding of the current study is that Gd(ABE-DTTA) has a similar ability already at an earlier age of infarct, i.e. 14 days following MI. It shows that the infarct affinity of Gd(ABEDTTA) has already vanished in the subacute phase of scar healing. This study also confirms the agent's ability to differentiate acute and older myocardial infarcts in an experimental study design different from the design in the previous study. It is also demonstrated, that $\mathrm{T} 2 \mathrm{w}$ imaging highlights the infarcts and the segments supplied by the infarct-related artery ("area at risk") similarly in the acute, subacute and late subacute phase, i.e. T2w imaging is not able to distinguish among these different phases of the myocardial infarct healing during the time window the study uses while Gd(ABE-DTTA) does, using the animal model, phased-array coil, and pulse sequences described above.

To the best of our knowledge, similar results have not been obtained using any other MRI contrast agent or any other MRI method, capable of differentiating new myocardial infarcts from existing acute infarcts as early as in the subacute phase, achieving this with high sensitivity and specificity.

Several methods using cardiovascular MRI for differentiation between acute and older myocardial infarcts have been published, but none of them showed ability to differentiate an acute infarct from a subacute infarct, allowing its use for infarct age differentiation early on.

An intravascular, high molecular weight contrast agent, P792, Vistarem (Guerbet Group, Paris, France) was recently studied by Saeed et al. [17] at two different time-points in a single MI, in a porcine model. Vistarem induced hyperenhancement in the acute infarct but not in the later, chronic phase of MI. The authors have also demonstrated the superiority of their method over the T2-weighted (T2w) MRI technique for differentiation between acute and chronic MI. The significant difference from our current study was that Saeed et al. elucidated the affinity to MI of the CA in an advanced, chronic phase of scar healing, at 2 months of infarct age.

Similarly, Abdel-Aty et al. [18] compared patients' MI in the acute vs. chronic phase. They combined Gd(DTPA)-induced DE with T2-weighted (T2w) MRI. T2-weighted MRI sequences are sensitive to water-bound protons. The distinction was based on an elevated $\mathrm{T} 2 \mathrm{w}$ signal intensity due to infarct-related edema, which was expected to be present exclusively in the acute phase of MI [18]. Abdel-Aty et al. applied their method to compare with an older infarct than was investigated in our current study.

Kim et al. [19] used contrast enhanced steady state free precession (SSFP) MRI for the differentiation between acute and chronic (above 6 month old) MIs in patients. Two minutes after Gd(DTPA) administration they observed elevated mean signal intensity in acute infarct areas with SSFP MRI. Chronic infarct regions, however, showed signal intensities similar to that of normal myocardium. 
Our results showing that $\mathrm{T} 2 \mathrm{w}$ imaging enhances the signal intensity in the territory of myocardial infarcts in subacute and late subacute infarcts are in accord with a previous publication of Johnstone et al. [20]. They found that the T2 relaxation time of the infarcted myocardium increased markedly at 3 days and remained elevated for 2 months in rabbits.

A 14 days old infarct is markedly different from a 28 days old infarct from the histological point of view. Richard et al. evaluated 14 day old myocardial infarcts [21] in a canine, reperfused model which were "characterized by a central core of persistent necrotic myocytes surrounded by an irregular rim of inflammatory cells, macrophages, and young scar (granulation tissue) composed of fibroblasts, new capillaries, and collagen." Histology samples taken from four week old myocardial infarcts of canines [5, 6], on the other hand, showed a more advanced stage of healing, with granulation tissue, collagen deposition, and small areas of interstitial fibrosis adjacent to the late subacute infarct. It is clear from these tissue differences that no extrapolations could be made about the affinity of Gd(ABEDTTA) to subacute infarct on the basis of results obtained in late subacute infarct, and that experimental comparison between the two was warranted. It also needs to be noted, from a potential clinical point of view, that MRI distinction between an acute and a subacute infarct would be of paramount importance for the in vivo validation of a pathological and clinical entity, the myocardial infarct extension. Infarct extension is defined clinically as an early inhospital reinfarction after myocardial infarction, appearing in histology as acute foci of contraction band necrosis around a subacute infarct [22]. Until now, the in vivo verification of this pathological entity has not been possible with any imaging modality due to the lack of capability for differentiation between an acute and a subacute infarct.

The exact mechanism of the DE phenomenon is not known to date even for standard extracellular agents. Enlarged distribution volume due to sarcolemmal membrane rupture in acute infarcts and low grade of cellularity with expanded interstitial collagen matrix in chronic scar tissue have been suggested as potential mechanisms for infarct accumulation of such agents [2, 23-26]. The exact mechanism of the delayed enhancement effect induced by Gd(ABE-DTTA) is not known to date, either. The possible mechanisms [5, 6] may differ from that induced by standard extracellular CAs considering the different behaviour of the two agent types in subacute infarcts.

The differentiation shown by Gd(ABE-DTTA) on the basis of infarct age as early as in the subacute phase, indicates that this agent may become a reliable diagnostic tool in several important clinical situations, once it is approved for human application. The most important potential scenarios would be the following:

\section{Localization of the "culprit" vessel in patients with multi-vessel CAD in acute NSTEMI in the presence of an old MI}

In the course of a non-STEMI in patients with multi-vessel CAD, the determination of the culprit vessel could be challenging if the ECG is non-informative. In these cases, DE-MRI with standard extracellular contrast agents (e.g. Gd(DTPA)) can localize the myocardial infarct (NSTEMI). If an older infarct is also present, Gd(DTPA) will highlight both the old and the new infarct. The additional use of Gd(ABE-DTTA) could selectively highlight the 
infarct corresponding with the culprit vessel. This could serve as a guide to the interventionalist for PCI.

\section{Detection of the extension of the myocardial infarct in its subacute phase}

Infarct extension is an event in the course of myocardial infarction [27] with serious short and long term consequences. It is defined clinically as an early in-hospital reinfarction after myocardial infarction. The pathologic finding of infarct extension is necrotic and healing myocardium of different recent (acute and subacute) ages within the same infarct territory [22]. Until now, the in vivo verification of this pathological event was not possible with any imaging modality due to the lack of capability for differentiation between an acute and a subacute infarct. Gd(ABE-DTTA), however, would be able to distinguish between acute and subacute myocardial infarcts allowing the in vivo verification of extension of the myocardial infarct.

\section{Study limitations}

Our study has some limitations. Because of its special tissue kinetics properties, it is necessary to administer Gd(ABE-DTTA) ca. $24 \mathrm{~h}$ before MRI imaging. This would introduce an inconvenience, but not an unprecedented one, in a clinical setting.

\section{Conclusions}

In summary, we have shown that DE-MRI using Gd(ABE-DTTA) differentiates with high sensitivity and specificity between acute and 2-week-old (subacute) MI as it does between acute and 4-week old (late subacute) MI, in a reperfused, canine, single MI model. The infarct affinity of Gd(ABE-DTTA) vanishes as early as the subacute phase of scar healing.

This feature of Gd(ABE-DTTA) may become a reliable tool in several clinical situations, assuming the agent's approval for human application.

\section{Acknowledgments}

This study was partially supported by National Institutes of Health; Grant Number: 1R41 HL084844. An abstract of this work was presented at the 38th Annual Meeting of the North American Society of Cardiovascular Imaging (NASCI), October 3-5, 2010 Seattle, Washington. A part of this work (the clinical implications paragraphs in the Discussion section) was presented at the 59th Annual Scientific Session of the American College of Cardiology, March 2010, Atlanta, Georgia.

\section{References}

1. Setser R, Bexell D, O’Donnell T, Stillman A, Lieber M, Schoenhagen P, White R. Quantitative assessment of myocardial scar in delayed enhancement magnetic resonance imaging. J Magn Reson Imaging. 2003; 18(4):434-441. [PubMed: 14508780]

2. Kim RJ, Fieno DS, Parrish TB, Harris K, Chen EL, Simonetti O, Bundy J, Finn JP, Klocke FJ, Judd RM. Relationship of MRI delayed contrast enhancement to irreversible injury, infarct age, and contractile function. Circulation. 1999; 100(19):1992-2002. [PubMed: 10556226]

3. Kim RJ, Wu E, Rafael A, Chen EL, Parker MA, Simonetti O, Klocke FJ, Bonow RO, Judd RM. The use of contrast-enhanced magnetic resonance imaging to identify reversible myocardial dysfunction. N Engl J Med. 2000; 343(20):1445-1453. [PubMed: 11078769]

4. Fieno DS, Hillenbrand HB, Rehwald WG, Harris KR, Decker RS, Parker MA, Klocke FJ, Kim RJ, Judd RM. Infarct resorption, compensatory hypertrophy, and differing patterns of ventricular 
remodeling following myocardial infarctions of varying size. J Am Coll Cardiol. 2004; 43(11): 2124-2131. [PubMed: 15172424]

5. Kirschner R, Varga-Szemes A, Toth L, Simor T, Suranyi P, Ruzsics B, Kiss P, Toth A, Baker R, Brott BC, Litovsky S, Elgavish A, Elgavish GA. Reinfarction-specific magnetic resonance imaging contrast agent. J Am Coll Cardiol. 2010; 55(Supplement 1)(10):A84.E795-A784.E795.

6. Kirschner R, Toth L, Varga-Szemes A, Simor T, Suranyi P, Kiss P, Ruzsics B, Toth A, Baker R, Brott B, Litovsky S, Elgavish A, Elgavish G. Differentiation of acute and four-week old myocardial infarct with Gd(ABE-DTTA)-enhanced CMR. J Cardiovasc Mag Reson. 2010; 12(1):22.

7. Ruzsics B, Suranyi P, Kiss P, Brott BC, Elgavish A, Saab-Ismail NH, Simor T, Elgavish GA. Gd(ABE-DTTA), a novel contrast agent, at the MRI-effective dose shows absence of deleterious physiological effects in dogs. Pharmacology. 2006; 77(4):188-194. [PubMed: 16877874]

8. Suranyi P, Kiss P, Ruzsics B, Brott BC, Simor T, Elgavish A, Baker RA, Saab-Ismail NH, Elgavish GA. In vivo myocardial tissue kinetics of Gd(ABE-DTTA), a tissue-persistent contrast agent. Magn Reson Med. 2007; 58(1):55-64. [PubMed: 17659616]

9. Suranyi P, Kiss P, Brott BC, Simor T, Elgavish A, Ruzsics B, Saab-Ismail NH, Elgavish GA. Percent infarct mapping: an R1-map-based CE-MRI method for determining myocardial viability distribution. Magn Reson Med. 2006; 56(3):535-545. [PubMed: 16892184]

10. Ruzsics B, Suranyi P, Kiss P, Brott BC, Elgavish A, Simor T, Elgavish GA. Head-to-head comparison between delayed enhancement and percent infarct mapping for assessment of myocardial infarct size in a canine model. J Magn Reson Imaging. 2008; 28(6):1386-1392. [PubMed: 19025946]

11. Saab-Ismail NH, Simor T, Gaszner B, Lorand T, Szollosy M, Elgavish GA. Synthesis and in vivo evaluation of new contrast agents for cardiac MRI. J Med Chem. 1999; 42(15):2852-2861. [PubMed: 10425094]

12. Beek AM, Bondarenko O, Afsharzada F, van Rossum AC. Quantification of late gadolinium enhanced CMR in viability assessment in chronic ischemic heart disease: a comparison to functional outcome. J Cardiovasc Magn Reson. 2009; 11(1):6. [PubMed: 19272147]

13. Friedrich MG, Abdel-Aty H, Taylor A, Schulz-Menger J, Messroghli D, Dietz R. The salvaged area at risk in reperfused acute myocardial infarction as visualized by cardiovascular magnetic resonance. J Am Coll Cardiol. 2008; 51(16):1581-1587. [PubMed: 18420102]

14. Schwitter J, Saeed M, Wendland MF, Derugin N, Canet E, Brasch RC, Higgins CB. Influence of severity of myocardial injury on distribution of macromolecules: extravascular versus intravascular gadolinium-based magnetic resonance contrast agents. J Am Coll Cardiol. 1997; 30(4):1086-1094. [PubMed: 9316544]

15. Simonetti OP, Kim RJ, Fieno DS, Hillenbrand HB, Wu E, Bundy JM, Finn JP, Judd RM. An improved MR imaging technique for the visualization of myocardial infarction. Radiology. 2001; 218(1):215-223. [PubMed: 11152805]

16. Witte, RS.; Witte, JS. Statistics. 5th edn edn.. Fort Worth: Harcourt Brace College Publishers; 1997.

17. Saeed M, Weber O, Lee R, Do L, Martin A, Saloner D, Ursell P, Robert P, Corot C, Higgins CB. Discrimination of myocardial acute and chronic (scar) infarctions on delayed contrast enhanced magnetic resonance imaging with intravascular magnetic resonance contrast media. J Am Coll Cardiol. 2006; 48(10):1961-1968. [PubMed: 17112985]

18. Abdel-Aty H, Zagrosek A, Schulz-Menger J, Taylor AJ, Messroghli D, Kumar A, Gross M, Dietz R, Friedrich MG. Delayed enhancement and T2-weighted cardiovascular magnetic resonance imaging differentiate acute from chronic myocardial infarction. Circulation. 2004; 109(20):24112416. [PubMed: 15123531]

19. Kim KA, Seo JB, Do KH, Heo JN, Lee YK, Song JW, Lee JS, Song KS, Lim TH. Differentiation of recently infarcted myocardium from chronic myocardial scar: the value of contrast-enhanced SSFP-based cine MR imaging. Korean J Radiol. 2006; 7(1):14-19. [PubMed: 16549951]

20. Johnston D, Homma S, Liu P, Weilbaecher D, Rokey R, Brady T, Okada R. Serial changes in nuclear magnetic resonance relaxation times after myocardial infarction in the rabbit: relationship to water content, severity of ischemia, and histopathology over a six-month period. Magn Reson Med. 1988; 8(4):363-379. [PubMed: 3231066] 
21. Richard V, Murry CE, Reimer KA. Healing of myocardial infarcts in dogs. Effects of late reperfusion. Circulation. 1995; 92(7):1891-1901. [PubMed: 7671374]

22. Hutchins GM, Bulkley BH. Infarct expansion versus extension: two different complications of acute myocardial infarction. Am J Cardiol. 1978; 41(7):1127-1132. [PubMed: 665522]

23. Kim RJ, Chen EL, Lima JA, Judd RM. Myocardial Gd-DTPA kinetics determine MRI contrast enhancement and reflect the extent and severity of myocardial injury after acute reperfused infarction. Circulation. 1996; 94(12):3318-3326. [PubMed: 8989146]

24. Mahrholdt H, Wagner A, Holly TA, Elliott MD, Bonow RO, Kim RJ, Judd RM. Reproducibility of chronic infarct size measurement by contrast-enhanced magnetic resonance imaging. Circulation. 2002; 106(18):2322-2327. [PubMed: 12403661]

25. Choi KM, Kim RJ, Gubernikoff G, Vargas JD, Parker M, Judd RM. Transmural extent of acute myocardial infarction predicts long-term improvement in contractile function. Circulation. 2001; 104(10):1101-1107. [PubMed: 11535563]

26. Fieno DS, Kim RJ, Chen EL, Lomasney JW, Klocke FJ, Judd RM. Contrast-enhanced magnetic resonance imaging of myocardium at risk: distinction between reversible and irreversible injury throughout infarct healing. J Am Coll Cardiol. 2000; 36(6):1985-1991. [PubMed: 11092675]

27. Weisman HF, Healy B. Myocardial infarct expansion, infarct extension, and reinfarction: Pathophysiologic concepts. Prog Cardiovasc Dis. 1987; 30(2):73-110. [PubMed: 2888158] 
$\leftarrow$ Day 28 - MRI session: Gd(ABE-DTTA)-enhanced DE images with IR-GRE sequence, TTC

$\leftarrow$ Day 27 - MRI session: IR-GRE MRI without CA $\rightarrow$ if there is enhancement, exclude animal, else do T2w images followed by Gd(DTPA)-enhanced DE MRI, then give Gd(ABE-DTTA)

$\leftarrow$ Day 14 -MRI session: Gd(ABE-DTTA)-enhanced DE images with IR-GRE sequence

$\leftarrow$ Day $13-$ MRI session: IR-GRE images w/o CA $\rightarrow$ if there is enhancement in infarct, exclude animal from Day 14, else do T2w images and then give Gd(ABE-DTTA)

$\leftarrow$ Day 4 - MRI Session: Gd(ABE-DTTA)-enhanced DE Images with IR-GRE sequence

$\leftarrow$ Day 3 - MRI Session: T2w images, Administration of Gd(ABE-DTTA)

$\leftarrow$ Day 0 - Infarct generation: LAD occlusion $180 \mathrm{~min}$

Fig. 1.

Experimental design and timeline of events 


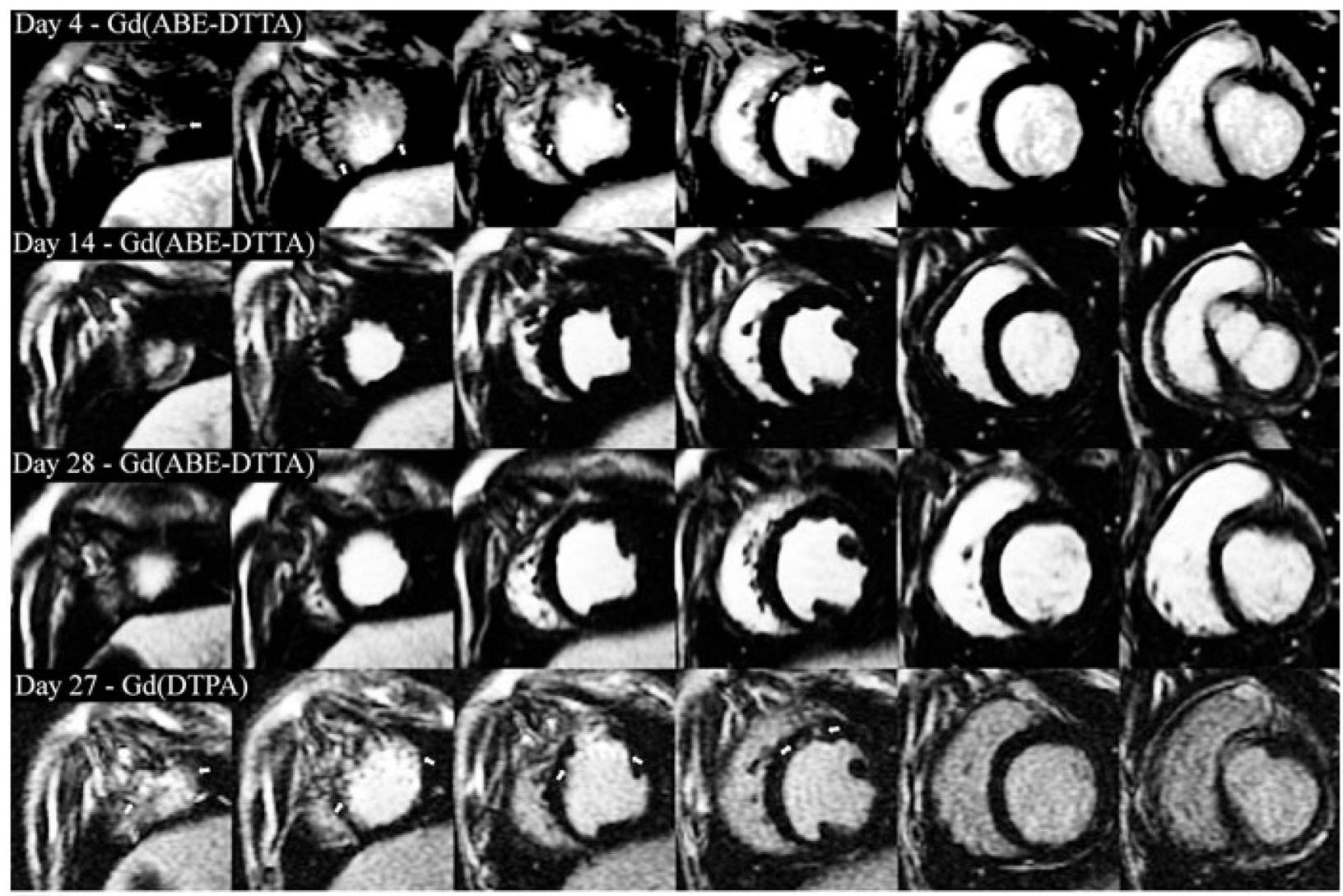

TTC
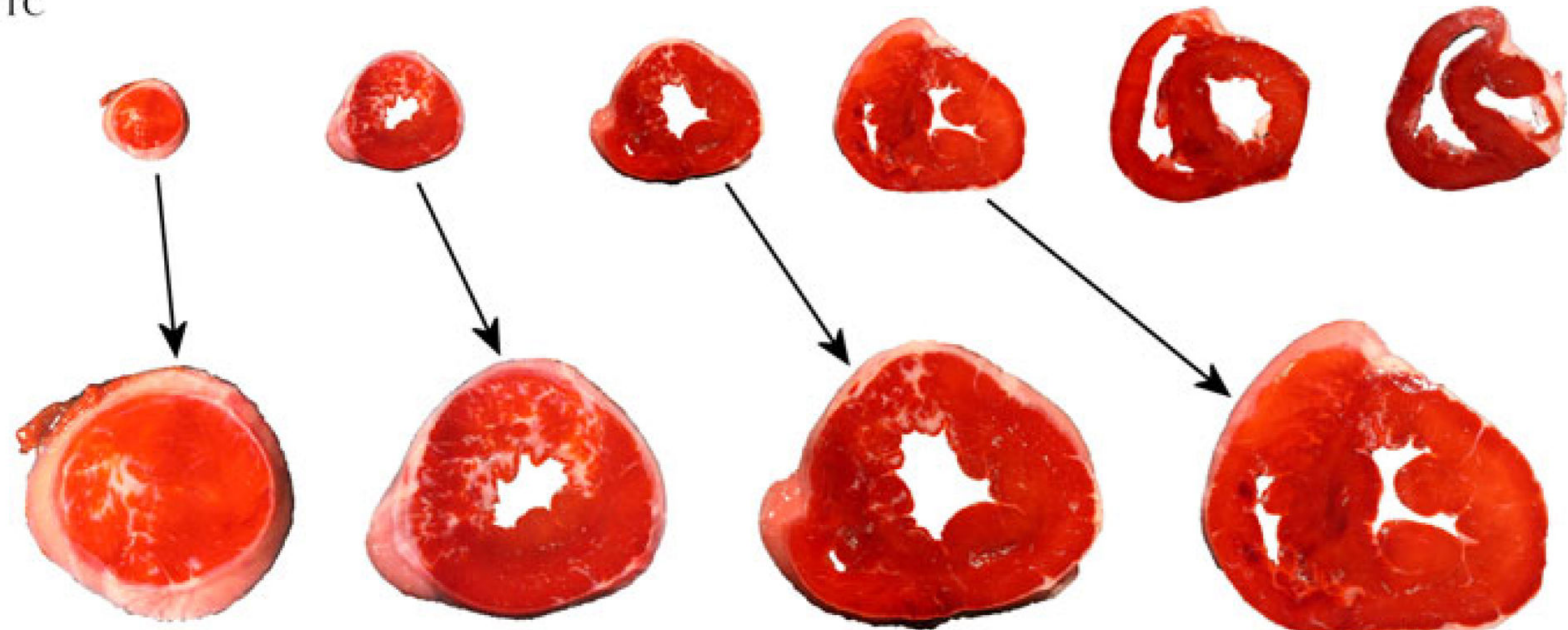

Fig. 2.

Differentiation between acute vs. subacute myocardial infarctions. DE images taken in the presence of Gd(ABE-DTTA) on days 4, 14, and 28 after MI (panels 1 through 3 from top, respectively). Gd(ABE-DTTA) highlights the infarct on day 4 (see arrows), but does not on days 14 and 28. DE image set with Gd(DTPA) on day 27 (panel 4 from top). This image set ascertains that infarct is present (see arrows) despite the fact that it is not highlighted by Gd(ABE-DTTA) anymore. Corresponding TTC slices (panel 5 from top, and with increased size in panel 6 from top) 


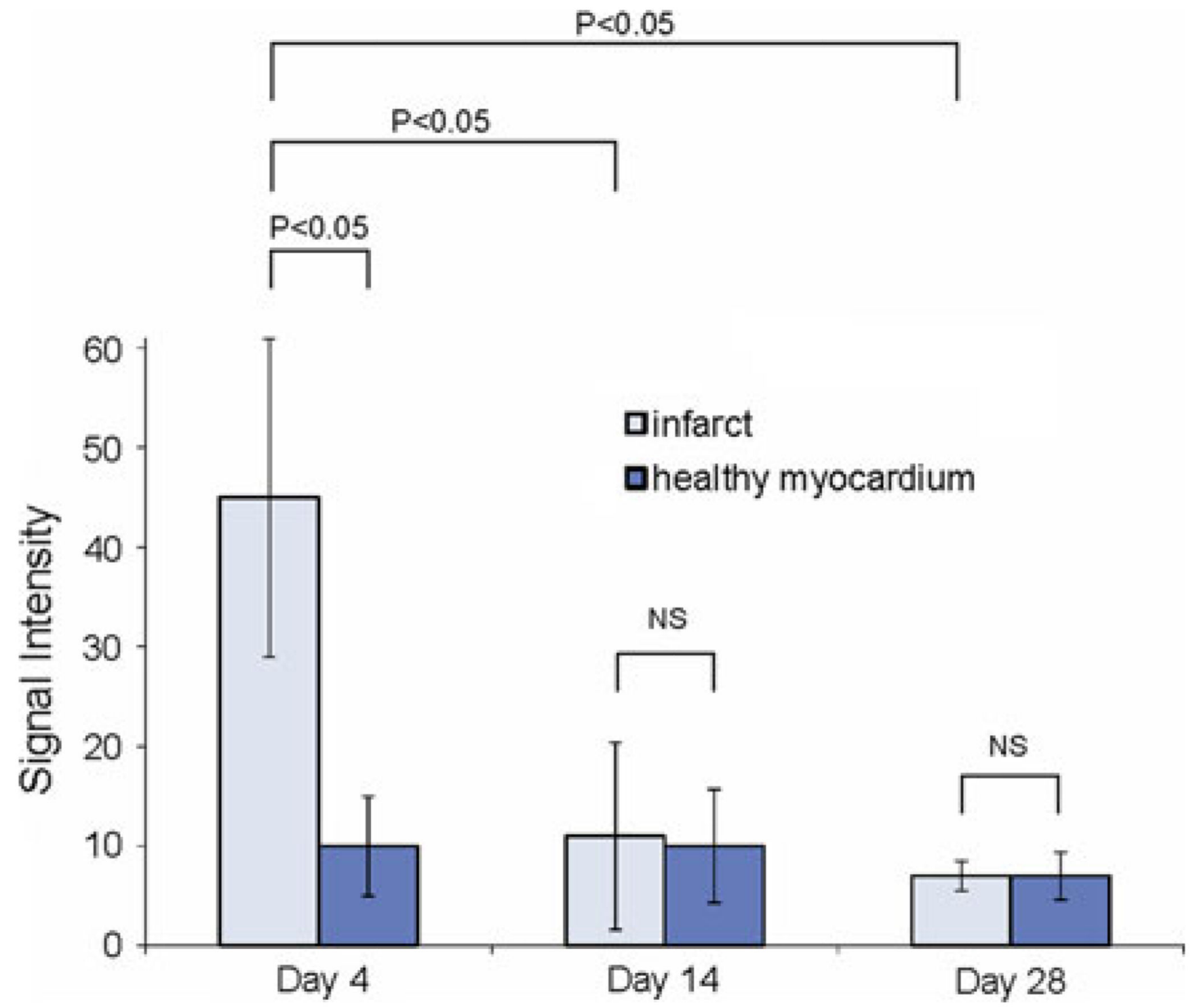

Fig. 3.

Comparison of signal intensity values at different infarct ages. Mean $(n=8)$ Signal intensity values induced by Gd(ABE-DTTA) in areas of infarcts (bright blue bars) versus healthy myocardium (dark blue bars) at different infarct ages. $P$ values pertain to pairwise comparisons by the Holm-Sidak method of the six different experimental subgroups 


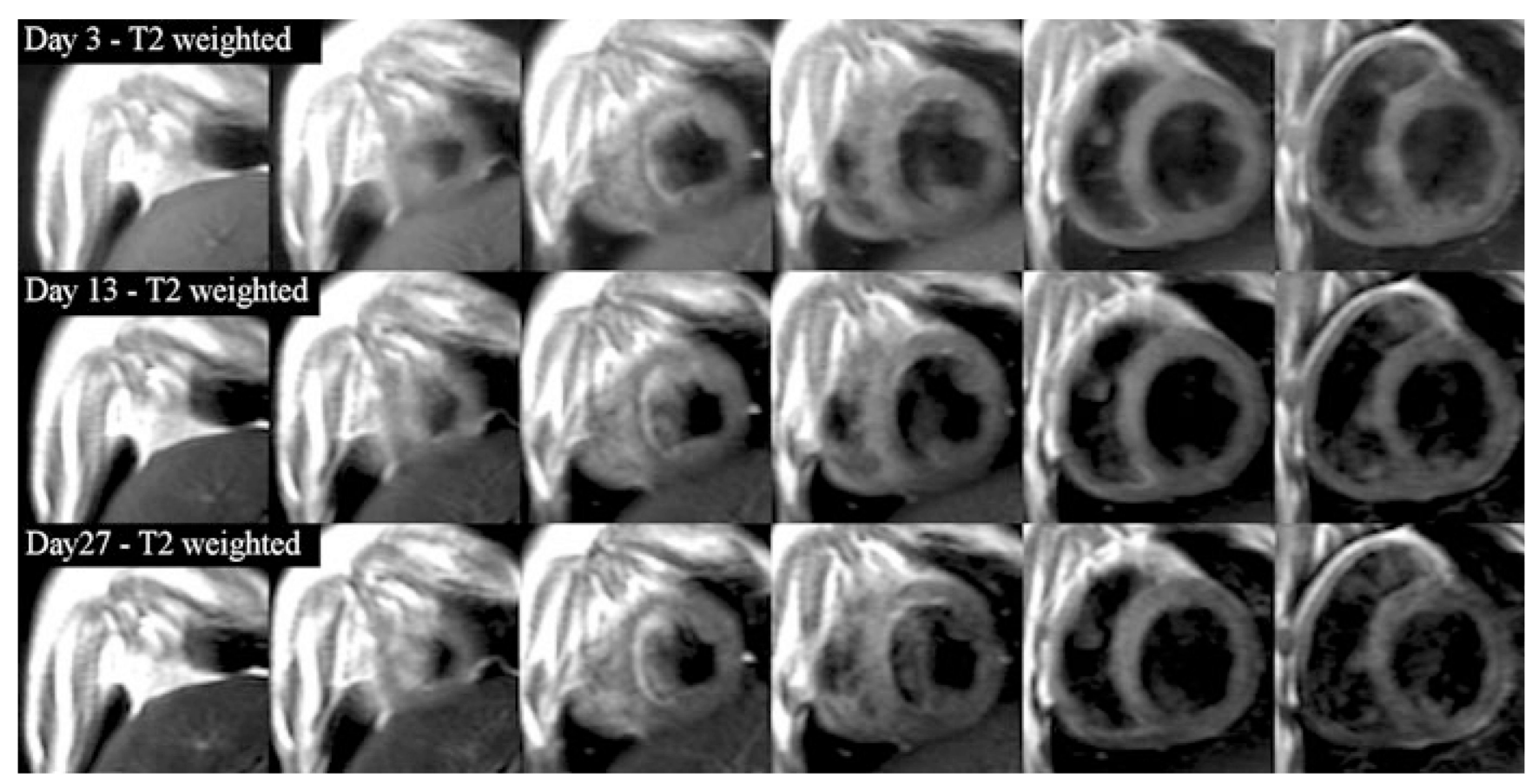

Fig. 4.

$\mathrm{T} 2 \mathrm{w}$ images. Corresponding $\mathrm{T} 2$ weighted black-blood spin-echo images ( $\mathrm{TE}=60 \mathrm{~ms}$ ) of the same dog on Fig. 3 taken on days 3, 13, and 27 post MI (panels 1 through 3 from top, respectively). Note, that $\mathrm{T} 2 \mathrm{w}$ signal enhancement can be observed in the anterior and anteroseptal segments not only during the acute but also during the subacute and late subacute phases of the myocardial infarct. The entire LAD-supply area (infarct related artery) is enhanced (area at risk), and not only the infarct- containing slices are involved. The pattern of the $\mathrm{T} 2 \mathrm{w}$-enhancement is quite the same in the 3 different phases of infarct scar healing 